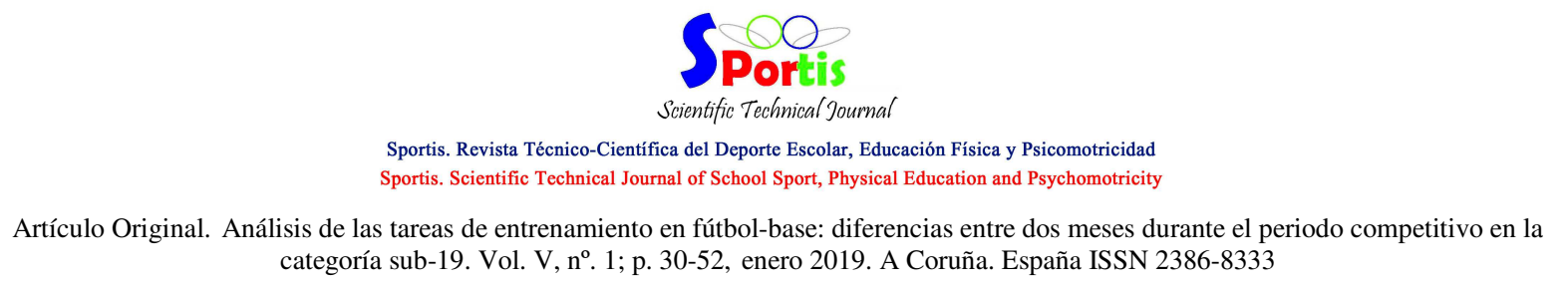

\title{
Análisis de las tareas de entrenamiento en fútbol-base: diferencias entre dos meses durante el periodo competitivo en la categoría sub-19
}

\section{Analysis of training task in grassroots: differences between two months during the competitive period in under-19 football players}

\author{
Gamonales Puerto, J.M. ${ }^{1}$; Gómez Carmona, C.D. ${ }^{1,2}$; León Guzmán, K. ${ }^{1,3}$; García Santos, D. ; \\ Gamero Portillo, M.G. ${ }^{1}$; Muñoz Jiménez, J. ${ }^{1,3}$ \\ ${ }^{1}$ Universidad de Extremadura, Cáceres, España. \\ ${ }^{2}$ Universidad de Murcia, San Javier, España. \\ ${ }^{3}$ Universidad Autónoma de Chile, Chile.
}

Autor de correspondencia: José Martín Gamonales Puerto. Contacto: mgamonales@unex.es

Cronograma editorial: Artículo recibido: 28/05/2018 Aceptado: 26/09/2018 Publicado: 01/01/2019

DOI: https://doi.org/10.17979/sportis.2019.5.1.3469

Financiación: Este trabajo ha sido parcialmente subvencionado por la Ayuda a los Grupos de Investigación (GR15122) de la Junta de Extremadura (Consejería de Empleo e Infraestructuras); con la aportación de la Unión Europea a través de los Fondos Europeos de Desarrollo Regional (FEDER).

Conflicto de intereses: Trabajo desarrollado dentro del Grupo de Optimización del Entrenamiento y Rendimiento Deportivo (GOERD) de la Facultad de Ciencias del Deporte de la Universidad de Extremadura. Todos los autores hemos contribuido en la realización del manuscrito y certificamos que no ha sido publicado ni está en vías de consideración para su publicación en otra revista. Aceptamos las normas de publicación de la Revista. Asimismo, transferimos los derechos del presente trabajo a Sportis: Scientific Journal of School Sport, Physical Education and Psychomotricity con ISBN 2386-8333, firmado por todos los autores.

\section{Resumen}

Actualmente, existe una falta de categorización en relación al diseño de las tareas de entrenamiento en el fútbol-base, y más concretamente en la categoría sub-19. La herramienta SIATE es un instrumento objetivo y útil para el registro de las tareas, que únicamente ha sido utilizado en una investigación previa en el contexto del fútbol. Por tanto, el objetivo del presente estudio fue analizar y comparar las características pedagógicas de las tareas de entrenamiento diseñadas durante un periodo competitivo de dos meses de duración en categoría sub-19. Se analizaron un total de 114 tareas de entrenamiento repartidas en 17 sesiones durante dos meses, enero $(n=9)$ y febrero $(n=8)$. El instrumento empleado para el análisis de las tareas fue el SIATE, donde se analizaron las siguientes variables pedagógicas: (a) Situación de juego; (b) Presencia de portero; (c) Fase de juego; (d) Tipo de contenido; (e) Medio de iniciación deportiva; (f) Nivel de oposición y (g) Línea de juego. Los resultados

\footnotetext{
Para citar este artículo utilice la siguiente referencia: Gamonales Puerto, J.M.; Gómez Carmona, C.D.; León Guzmán, K.; García Santos, D.; Gamero Portillo, M.G.; Muñoz Jiménez, J. (2019). Análisis de las tareas de entrenamiento en fútbol-base: diferencias entre dos meses durante el periodo competitivo en la categoría sub-19. SportisSci J, 5 (1), 30-52. DOI: https://doi.org/10.17979/sportis.2019.5.1.3469

http://revistas.udc.es/
} 
Artículo Original. Análisis de las tareas de entrenamiento en fútbol-base: diferencias entre dos meses durante el periodo competitivo en la categoría sub-19. Vol. V, no. 1; p. 30-52, enero 2019. A Coruña. España ISSN 2386-8333

muestran en el mes de enero un predominio de juegos reducidos de 6vs6 sin presencia de portero. En cambio, en el segundo mes existen mayoritariamente juegos de 8vs7 y combinados en espacios reducidos sin portero. En cuanto a la comparativa entre meses de entrenamientos, se encuentran diferencias significativas en todas las variables pedagógicas. En conclusión, se encuentra un predominio del uso de juegos reducidos para el desarrollo de las capacidades físico-técnico-tácticas durante las sesiones de entrenamiento, siendo posible su registro de forma objetiva gracias a la herramienta SIATE. Existe una participación minoritaria del portero en las tareas, aspecto que debería ser corregido para la mejora del rendimiento y una mayor similitud a las situaciones de juego real durante la competición.

\title{
Palabras clave
}

SIATE; planificación; entrenamiento; deportes colectivos.

\begin{abstract}
Currently, a lack of research exists in relation to the categorization of training tasks in grassroots, and specially in under-19 category. The SIATE is an objective and useful tool for registering training tasks. This tool has been used only in a previous research in football context. Therefore, the aim of the present research is to analyze and compare the pedagogical characteristics of training tasks designed by a coach that worked in an under-19 team during a two-months competitive period. A total of 114 tasks, divided in 17 training sessions during two months, january $(n=9)$ and february $(n=8)$ were registered. The tool utilized to analyse the tasks was the SIATE, where the following pedagogical variables were analyzed (a) Game situations; (b) With or without goalkeeper; (c) Game phase; (d) Type of content; (e) Sports initiation medium; (f) Level of opposition and (g) Playing line. The main results show in the first month a prevalence of 6vs6 small-sided games without goalkeeper. Instead, in the second month, it is shown 8vs7 small-sided games and combined game situations without goalkeeper. In relation to the comparison analysis between months, statistical differences were found in all pedagogical variables. In conclusions, thus, in this u-19 team is found a prevalence of small-sided games to develop the physical-technical-tactical capacities of footballers during training sessions, being possible the objective register of them by the SIATE's tool. Besides, it exists a lack of goalkeeper participation in the training tasks that should be corrected to improve the performance and achieve a better simulation of real play context.
\end{abstract}

\section{Keywords}

SIATE; periodization; training; team sports. 


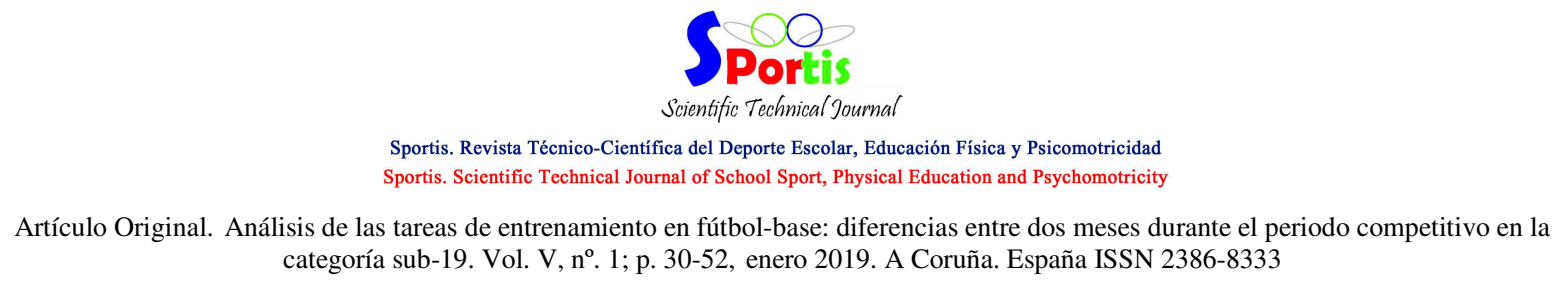
categoría sub-19. Vol. V, no. 1; p. 30-52, enero 2019. A Coruña. España ISSN 2386-8333

\section{Introducción}

La planificación de tareas de entrenamiento en los deportes de equipo ha evolucionado de manera significativa en relación a los métodos de enseñanza desde el uso de una metodología tradicional utilizada para la preparación en los deportes individuales (Arjol, 2012), compuestas por tareas inespecíficas, aisladas o globales específicas (Alarcón, Cárdenas, Miranda, Ureña y Piñar, 2010) hacia nuevos métodos de planificación como la periodización táctica (Leo-Marcos, Pulido-González, Sánchez-Oliva, Candela y García-Calvo, 2012) que son más específicos de la modalidad deportiva, con el objetivo de lograr jugadores con una mayor riqueza técnico-táctica. Esta nueva perspectiva es más flexible y adaptable a las necesidades del deportista donde el individuo debe construir su propio aprendizaje (Gil, Moreno, Claver, Moreno y Del Villar, 2016; González-Espinosa, Ibáñez, Feu y Galatti, 2017).

Esta metodología de entrenamiento se aleja de la utilización de tareas cerradas/analíticas orientadas a la repetición y reproducción, programando una práctica alternativa, abierta, en la que se utiliza como herramienta para el desarrollo de las capacidades físico-técnico-tácticas el juego modificado (Thorpe, Bunker y Almond, 1986), el cual presenta actualmente un interés creciente de estudio por parte de la comunidad científica y deportiva (Gracia, García-Rubio, Cañadas e Ibáñez, 2014; Hernández, Ortega y Palao, 2016). Por tanto, a través del uso de metodologías más activas como la enseñanza comprensiva, los entrenadores pueden utilizar un proceso de enseñanza-aprendizaje desde un punto de vista alternativo (Castejón, 2015), donde las tareas plantean un problema con la finalidad de que los jugadores sean conscientes de los aspectos técnico-tácticos a desarrollar en la tarea (Gray y Sproule, 2011), provocando una mayor percepción de dificultad durante su realización (García-Angulo y García-Angulo, 2018). El uso de tareas de entrenamiento basadas en el método comprensivo mejora significativamente la toma de decisiones individual y el comportamiento táctico global de los jugadores (García y Ruiz, 2003; Turner y Martinek, 1999).

\footnotetext{
Para citar este artículo utilice la siguiente referencia: Gamonales Puerto, J.M.; Gómez Carmona, C.D.; León Guzmán, K.; García Santos, D.; Gamero Portillo, M.G.; Muñoz Jiménez, J. (2019). Análisis de las tareas de entrenamiento en fútbol-base: diferencias entre dos meses durante el periodo competitivo en la categoría sub-19. SportisSci J, 5 (1), 30-52. DOI: https://doi.org/10.17979/sportis.2019.5.1.3469

http://revistas.udc.es/
} 
Artículo Original. Análisis de las tareas de entrenamiento en fútbol-base: diferencias entre dos meses durante el periodo competitivo en la categoría sub-19. Vol. V, nº. 1; p. 30-52, enero 2019. A Coruña. España ISSN 2386-8333

Para ello, previamente se requiere de la identificación de las exigencias de la competición para diseñar programas de entrenamiento más específicos para la modalidad deportiva (Torres-Ronda, Ric, Llabres-Torres, De las Heras y Del Alcazar, 2016). Las características específicas de la modalidad deportiva, así como las concepciones metodológicas de los entrenadores o técnicos deportivos en fútbol influyen en el desarrollo de los objetivos y la utilización de los contenidos deportivos, lo cual se plasma en las tareas de entrenamiento que son las unidades más concretas del proceso de planificación y programación del entrenamiento (Sáenz-López, Feu e Ibáñez, 2006), además de influir en factores psicológicos como la ansiedad que padecen los jugadores (González-Campos, 2016). Además de la metodología, otro factor influyente en el proceso de entrenamiento es la experiencia de los entrenadores que les permite diseñar, seleccionar y evolucionar las agrupaciones de las tareas deportivas durante el entrenamiento (Clemente, Martins y Mendes, 2015). Cuanto mayor es su experiencia más recursos disponen para afrontar esta competencia básica de todo entrenador (Ibáñez, Feu y Cañadas, 2016), donde es común reducir el número de jugadores y los espacios de juego (Sánchez-Sánchez, Yagüe, Fernández y Petisco, 2014). Por ello, la importancia del entrenamiento deportivo y de la idoneidad del proceso formativo es clave para un adecuado desarrollo y perfeccionamiento de los jugadores (Sáenz-López, Ibáñez, Giménez, Sierra y Sánchez, 2005; Sáenz-López, Jiménez, Giménez e Ibáñez, 2007; Cañadas, Ibáñez, Feu, García-Rubio y Parejo, 2011).

El análisis de las tareas de entrenamiento en el fútbol-base permitirá estudiar el posicionamiento metodológico, así como generar nuevas teorías de entrenamiento basadas en la práctica (Cañadas et al., 2011), convirtiéndose en una de las líneas emergentes para los científicos (Ibáñez et al, 2016). Estos trabajos permiten conocer las situaciones de juego empleadas por los entrenadores (Cañadas, Ibáñez, García, Parejo y Feu, 2013), los contenidos de entrenamiento (Cañadas e Ibáñez, 2010; Cañadas, Ibáñez y Leite, 2015), los medios de entrenamiento (Cañadas, Ibáñez, Feu, García-Rubio y Parejo, 2011; Cañadas, Parejo, Ibáñez, García-Rubio y Feu, 2009), las fases de juego (Cañadas, Ibáñez, García-Rubio, Parejo y Feu, 2012), la relación entre las variables pedagógicas del entrenamiento (Cañadas, Rodríguez, Feu, Parejo y García-Rubio, 2013; Gómez-Carmona, García-Rubio, Muñoz y Gamonales, 2018) o la carga durante una tarea (Gracia, García, Cañadas y Ibáñez, 2014) a lo largo de la temporada.

\footnotetext{
Para citar este artículo utilice la siguiente referencia: Gamonales Puerto, J.M.; Gómez Carmona, C.D.; León Guzmán, K.; García Santos, D.; Gamero Portillo, M.G.; Muñoz Jiménez, J. (2019). Análisis de las tareas de entrenamiento en fútbol-base: diferencias entre dos meses durante el periodo competitivo en la categoría sub-19. SportisSci J, 5 (1), 30-52. DOI: https://doi.org/10.17979/sportis.2019.5.1.3469

http://revistas.udc.es/
} 


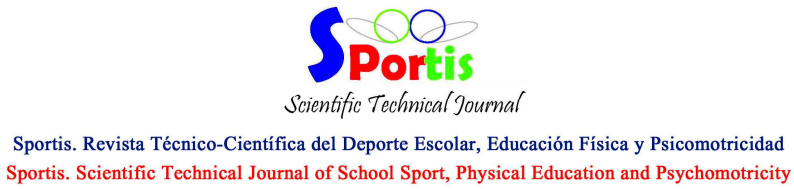

Sportis. Scientific Technical Journal of School Sport, Physical Education and Psychomotricity

Artículo Original. Análisis de las tareas de entrenamiento en fútbol-base: diferencias entre dos meses durante el periodo competitivo en la categoría sub-19. Vol. V, no $.1 ;$ p. 30-52, enero 2019. A Coruña. España ISSN 2386-8333

Además, el conocimiento de las tareas de entrenamiento aportará las claves para diseñar unos entrenamientos idóneos y adecuados que aseguren un trabajo donde se desarrollen variables que tengan que ver directa o indirectamente con los rasgos de la lógica interna del fútbol (Parlebas, 2001; Ortega-Toro y Sainz de Baranda, 2003) o durante la simulación del juego real (Casamichana, Castellano y Castagna, 2012; Hill-Haas, Dawson, Impellizzeri y Coutts, 2011), para la mejora de la toma de decisiones por parte del futbolista (Davids, Araujo, Correia y Vilar, 2013). Para su registro y posterior análisis, se han creado diferentes herramientas objetivas para la categorización de las tareas, entre las que se encuentra el instrumento denominado Sistema Integral para el Análisis de las Tareas de Entrenamiento (SIATE) (Ibañez et al. 2016). En la literatura científica, existe una única investigación que haya analizado las variables pedagógicas predominantes en las tareas de entrenamiento en el fútbol-base en categoría juvenil, encontrando un predominio del uso de los juegos reducidos (JR) sin la presencia de guardameta (Gómez-Carmona et al., 2018).

Por tanto, a partir de la bibliografía consultada, existe la necesidad de conocer las características de las tareas programadas durante las sesiones de entrenamiento en categoría juvenil y si la planificación de las mismas varía en diferentes periodos de la temporada, así como aumentar el conocimiento que complete el estado del arte en la investigación relacionada con el fútbol-base. Finalmente, los objetivos del presente estudio fueron: (a) caracterizar las tareas de entrenamiento diseñadas y desarrolladas por un entrenador de fútbolbase en la categoría juvenil y (b) comparar su planificación entre dos meses durante la temporada.

\section{Material y método}

\section{Diseño}

El diseño de la investigación se encuadra dentro de una estrategia descriptivo de tipo selectivo (Ato, López y Benavente, 2013). Se seleccionó un periodo de entrenamiento con una duración de dos meses. Las sesiones de entrenamiento fueron diseñadas y desarrolladas por un entrenador de fútbol-base, en la categoría juvenil, con la finalidad de conocer la planificación de tareas de entrenamiento.

\footnotetext{
Para citar este artículo utilice la siguiente referencia: Gamonales Puerto, J.M.; Gómez Carmona, C.D.; León Guzmán, K.; García Santos, D.; Gamero Portillo, M.G.; Muñoz Jiménez, J. (2019). Análisis de las tareas de entrenamiento en fútbol-base: diferencias entre dos meses durante el periodo competitivo en la categoría sub-19. SportisSci J, 5 (1), 30-52. DOI: https://doi.org/10.17979/sportis.2019.5.1.3469

http://revistas.udc.es/
} 


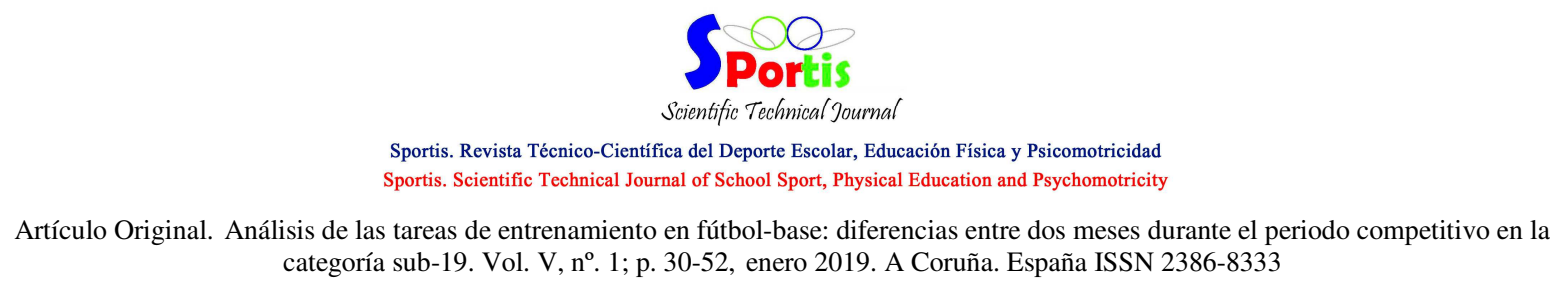
categoría sub-19. Vol. V, no. 1; p. 30-52, enero 2019. A Coruña. España ISSN 2386-8333

\section{Muestra}

Se analizaron 17 sesiones de correspondientes a dos meses de competición oficial durante la temporada 2017-2018, comprendiéndose entre los meses de enero y febrero. Durante el primer mes, se desarrollaron 9 sesiones y en el segundo mes se realizaron 8 sesiones. El equipo analizado de categoría sub-19 participaba en el campeonato regional de Liga de Primera División Extremeña Juvenil, organizado por la Federación Extremeña de Fútbol. El equipo entrenaba dos días por semana, martes y jueves. La duración de cada sesión fue de 1 hora y 30 minutos.

Durante las 17 sesiones de entrenamiento, se realizaron un total de 114 tareas las cuales fueron diseñadas, elaboradas y desarrolladas por un único entrenador, el cual presentaba una experiencia como jugador mayor de 25 años y como entrenador de 8 años. Además, posee como formación académica el Doctorado en Ciencias del Deporte y a nivel deportivo el título de Técnico Deportivo en Fútbol (Nivel II).

En la tabla 1, se muestra el tiempo empleado en las diferentes tareas planificadas por el entrenador a lo largo del periodo analizado, siendo este dividido en: (a) Tiempo total, que comprende la duración completa de la tarea; (b) Tiempo de explicación, es aquel destinado a la realización de las instrucciones pertinentes previa a la realización de la tarea, y (c) Tiempo útil que representa el tiempo efectivo de realización de la tarea donde se desarrollan las capacidades físico-técnico-tácticas del jugador.

Tabla $\mathrm{n}^{\circ}$ 1. Análisis descriptivo de la duración de las tareas analizadas (en minutos).

\begin{tabular}{|c|c|c|c|c|c|}
\hline \multirow{2}{*}{$\begin{array}{c}\text { Mes de } \\
\text { entrenamiento }\end{array}$} & & \multirow{2}{*}{ Tareas } & \multicolumn{3}{|c|}{ Tipos de tiempo } \\
\hline & & & Tiempo total & Tiempo explicación & Tiempo útil \\
\hline \multirow{3}{*}{ Enero } & \multirow{3}{*}{67} & Media \pm DE & $9.66 \pm 6.01$ & $1.07 \pm 0.75$ & $8.59 \pm 5.88$ \\
\hline & & Máximo & 30.00 & 3.50 & 28.66 \\
\hline & & Mínimo & 2.33 & 0 & 2.33 \\
\hline \multirow{3}{*}{ Febrero } & \multirow{3}{*}{47} & Media \pm DE & $12.23 \pm 10.73$ & $0.98 \pm 1.62$ & $11.25 \pm 9.52$ \\
\hline & & Máximo & 56.00 & 10.00 & 46.00 \\
\hline & & Mínimo & 180 & 0 & 160 \\
\hline \multirow{3}{*}{ Total } & \multirow{3}{*}{114} & Media $\pm \mathrm{DE}$ & $10.71 \pm 8.35$ & $1.03 \pm 1.19$ & $9.68 \pm 7.66$ \\
\hline & & Máximo & 56.00 & 10.00 & 46.00 \\
\hline & & Mínimo & 2.33 & 0 & 2.33 \\
\hline
\end{tabular}

DE: Desviación estándar

Para citar este artículo utilice la siguiente referencia: Gamonales Puerto, J.M.; Gómez Carmona, C.D.; León Guzmán, K.; García Santos, D.; Gamero Portillo, M.G.; Muñoz Jiménez, J. (2019). Análisis de las tareas de entrenamiento en fútbol-base: diferencias entre dos meses durante el periodo competitivo en la categoría sub-19. SportisSci J, 5 (1), 30-52. DOI: https://doi.org/10.17979/sportis.2019.5.1.3469

http://revistas.udc.es/ 


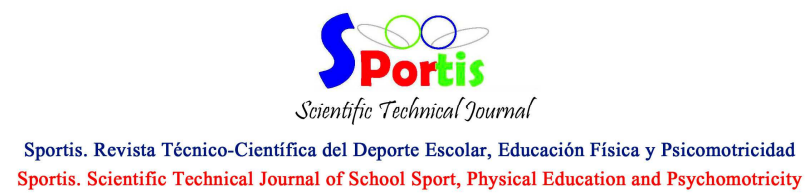

Artículo Original. Análisis de las tareas de entrenamiento en fútbol-base: diferencias entre dos meses durante el periodo competitivo en la categoría sub-19. Vol. V, nº. 1; p. 30-52, enero 2019. A Coruña. España ISSN 2386-8333

\section{Instrumento y variables analizadas}

Las variables empleadas en esta investigación son las habituales de los estudios recientes realizados en el fútbol, y se definieron de forma precisa por Ibáñez et al., (2016). Para ello, se utilizó la totalidad de las variables pedagógicas del instrumento SIATE, sistema metodológico para registrar y analizar los diferentes factores que inciden en el entrenamiento deportivo en deportes de invasión. Es una herramienta modulable desde el momento en el que se pueden definir la cantidad de información que los entrenadores registren de cada tarea de entrenamiento. Por tanto, cuanto mayor información se consiga obtener de cada tarea de entrenamiento más profundo podrá ser el análisis y la evaluación del mismo (Ibáñez et al., 2016). Por ello, la variable Línea de juego se adaptó a los puestos específicos del fútbol.

La variable independiente de la investigación es el Mes de entrenamiento. Todas las variables del estudio se categorizaron de forma numérica, con la finalidad de facilitar su registro y posterior análisis estadístico. En la figura 1, se muestran las variables pedagógicas del SIATE registradas en el estudio.

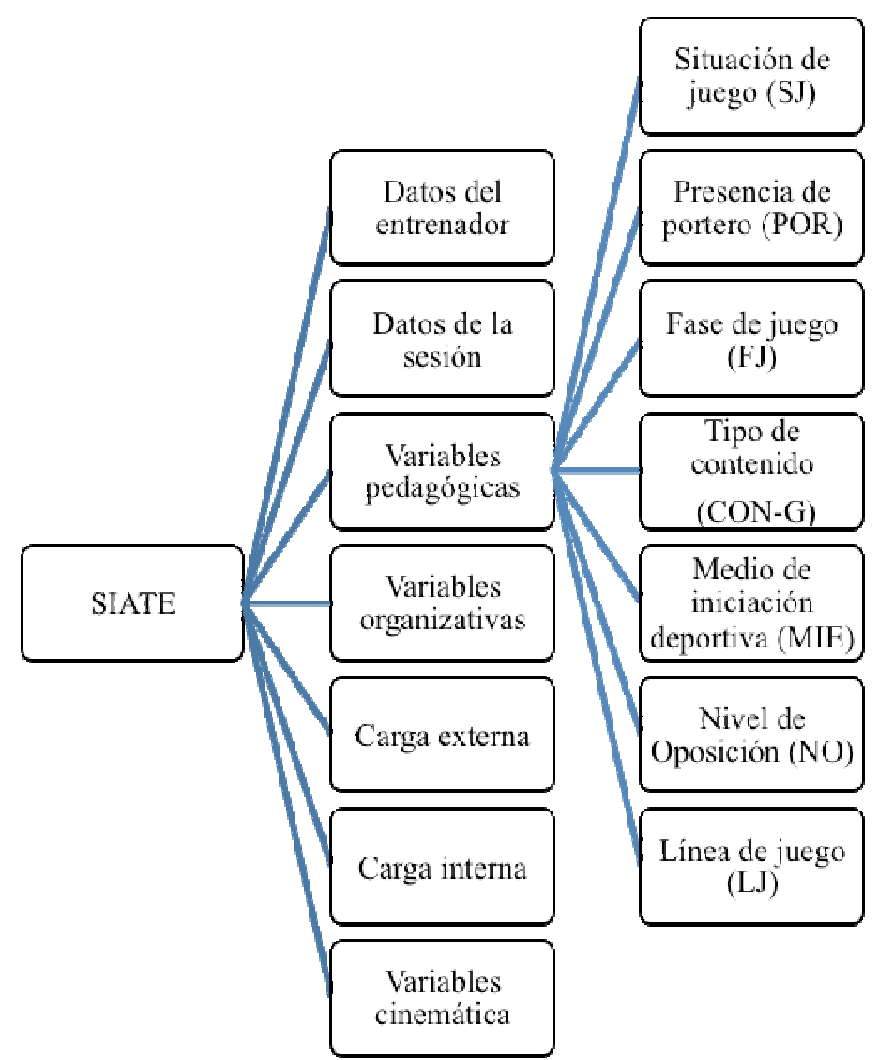

Figura $\mathrm{n}^{\circ}$ 1. Variables pedagógicas del instrumento SIATE registradas en la presente investigación.

Para citar este artículo utilice la siguiente referencia: Gamonales Puerto, J.M.; Gómez Carmona, C.D.; León Guzmán, K.; García Santos, D.; Gamero Portillo, M.G.; Muñoz Jiménez, J. (2019). Análisis de las tareas de entrenamiento en fútbol-base: diferencias entre dos meses durante el periodo competitivo en la categoría sub-19. SportisSci J, 5 (1), 30-52. DOI: https://doi.org/10.17979/sportis.2019.5.1.3469

http://revistas.udc.es/ 
Artículo Original. Análisis de las tareas de entrenamiento en fútbol-base: diferencias entre dos meses durante el periodo competitivo en la Procedimiento categoría sub-19. Vol. V, nº. 1; p. 30-52, enero 2019. A Coruña. España ISSN 2386-8333

Para conocer las tareas de entrenamiento diseñadas por un único entrenador de fútbolbase, en la categoría juvenil, durante el periodo competitivo, comprendido entre los meses de enero y febrero de 2018, así como para analizar las diferencias entre los meses seleccionados de forma aleatoria y por la facilidad de la muestra, se utilizó el instrumento SIATE. Herramienta útil, práctica y gratuita para analizar las tareas de entrenamiento en diferentes modalidades deportivas (Gómez-Carmona et al., 2018).

Se empleó el bloque de las variables pedagógicas. Para ello, previamente se formaron a dos observadores externos, para categorizar las tareas de entrenamiento. El proceso aplicado fue similar a los existentes en la literatura científica en otros contextos deportivos como en Fútbol a 5 para personas ciegas (Gamonales, Muñoz, León e Ibáñez, 2018) o en Rugby (Villarejo, Ortega, Gómez y Palao, 2014). Este proceso de formación y entrenamiento de observadores se dividió en cuatro etapas: etapa preparatoria; etapa de selección de los codificadores; etapa de formación de los observadores; y por último, etapa de confiabilidad. Se midió la fiabilidad inter-codificador mediante el índice Multirater Kappa free (Randolph, 2005), obteniéndose un valor mínimo de 0.89 en todas las variables analizadas. De esta manera, se estima el grado de precisión con el que se está midiendo las variables del estudio, pues la fiabilidad indica la consistencia del proceso de medición o de los resultados (Barraza, 2007). Estos resultados fueron similares e incluso superiores a los existente en la literatura científica relacionada con el Fútbol (Sarmento, Anguera, Campaniço y Leitão, 2010) o Fútbol a 5 para personas ciegas (Gamonales et al., 2018).

Posteriormente, tras el proceso de formación de los codificadores, los observadores registraron las tareas de entrenamiento. Por último, se creó la variable Mes de entrenamiento y se pasó a recoger, así como a tratar los datos del estudio. En la figura 2, se muestra el procedimiento llevado a cabo para la codificación de los observadores, así como el resto de fases del estudio de análisis de tareas de entrenamiento en el fútbol - base.

\footnotetext{
Para citar este artículo utilice la siguiente referencia: Gamonales Puerto, J.M.; Gómez Carmona, C.D.; León Guzmán, K.; García Santos, D.; Gamero Portillo, M.G.; Muñoz Jiménez, J. (2019). Análisis de las tareas de entrenamiento en fútbol-base: diferencias entre dos meses durante el periodo competitivo en la categoría sub-19. SportisSci J, 5 (1), 30-52. DOI: https://doi.org/10.17979/sportis.2019.5.1.3469

http://revistas.udc.es/
} 
Artículo Original. Análisis de las tareas de entrenamiento en fútbol-base: diferencias entre dos meses durante el periodo competitivo en la categoría sub-19. Vol. V, nº. 1; p. 30-52, enero 2019. A Coruña. España ISSN 2386-8333

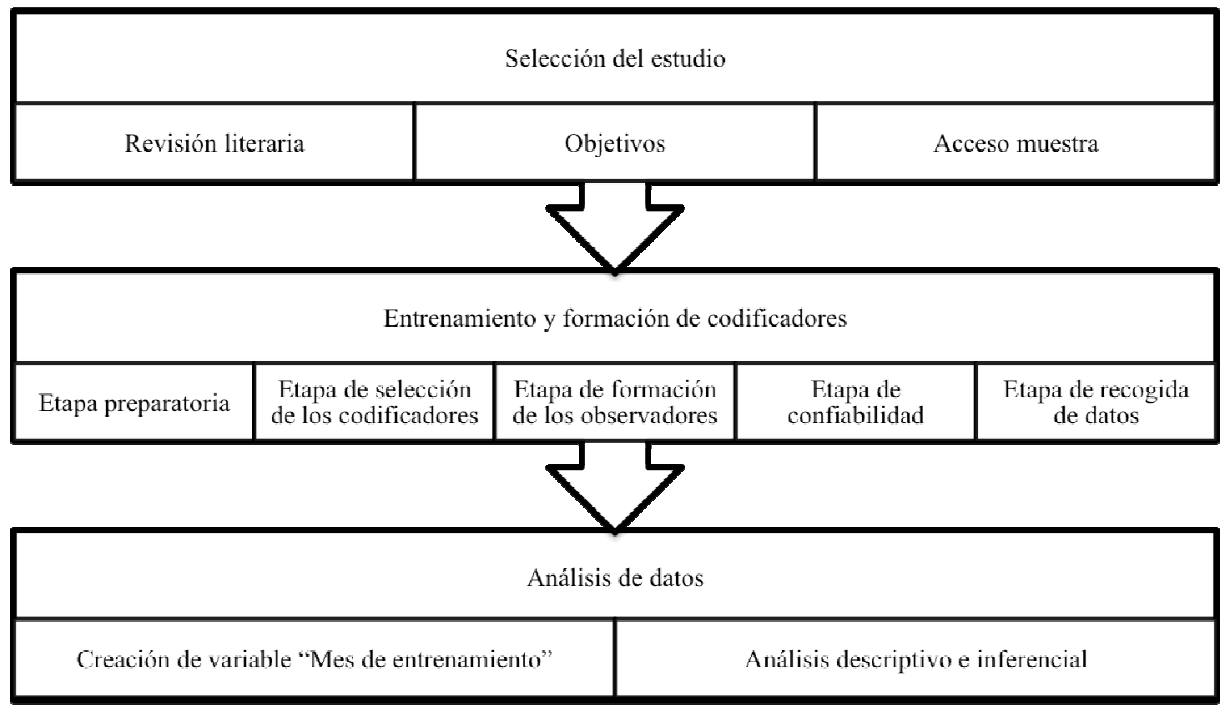

Figura $\mathrm{n}^{\circ}$ 2. Procedimiento del estudio de análisis de tareas de entrenamiento en el fútbol - base

\section{Análisis estadístico}

Para evaluar la fiabilidad de los datos recogidos por los codificadores, se llevó a cabo a través del procedimiento estadístico denominado Multirater Kappa free (Randolph, 2005), variante del coeficiente Kappa. Randolph (2005) señala que un valor de 0.70 o superior indica un alto grado de acuerdo entre codificadores. Este valor ha sido la medida de referencia adoptada para valorar la fiabilidad inter-codificador. Además, se calculó el promedio de la fiabilidad de los datos recogidos por los codificadores de forma parcial y total, con la finalidad de comprobar si el proceso de formación de los codificadores fue adecuado.

Tras el proceso de formación, así como de entrenamiento y confiabilidad entre observadores para el análisis de tareas de entrenamiento, y con los datos recogidos, se procedió a realizar un análisis descriptivo y de porcentajes del periodo competitivo analizado. Para conocer las diferencias entre los dos meses competitivos, se empleó Chi cuadrado $\left(\chi^{2}\right)$ y Coeficiente Phi $(\varphi c)$ de Cramer (Newell, Aitchison y Grant, 2014). El nivel de asociación del indicador $\varphi c$ de Cramer se interpreta a través de la propuesta de Crewson (2006). El grado de asociación entre las variables pedagógicas con la variable del estudio (Mes de entrenamiento) se realizó a través de los Residuos Tipificados Corregidos (RTC) de las tablas cruzadas (Field, 2009).

\footnotetext{
Para citar este artículo utilice la siguiente referencia: Gamonales Puerto, J.M.; Gómez Carmona, C.D.; León Guzmán, K.; García Santos, D.; Gamero Portillo, M.G.; Muñoz Jiménez, J. (2019). Análisis de las tareas de entrenamiento en fútbol-base: diferencias entre dos meses durante el periodo competitivo en la categoría sub-19. SportisSci J, 5 (1), 30-52. DOI: https://doi.org/10.17979/sportis.2019.5.1.3469

http://revistas.udc.es/
} 


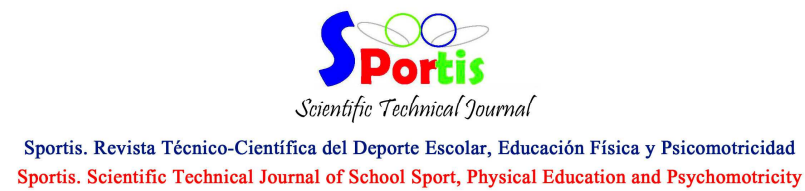

Sportis. Scientific Technical Journal of School Sport, Physical Education and Psychomotricity

Artículo Original. Análisis de las tareas de entrenamiento en fútbol-base: diferencias entre dos meses durante el periodo competitivo en la Resultados

categoría sub-19. Vol. V, nº. 1; p. 30-52, enero 2019. A Coruña. España ISSN 2386-8333

En la tabla 3, se muestran los resultados descriptivos de las variables del estudio en fútbol-base. En relación a las variables Situación de juego y Presencia de portero se encuentra un predominio de las tareas de $6 x 6$ en el Mes de enero (41.8\%), mientras que en el Mes de febrero se encuentra un mayor porcentaje en las Situaciones de $8 x 7$ (21.3\%) y Situaciones combinadas (21.3\%), existiendo en ambos meses un mayor número de tareas sin la presencia de portero (enero 83.6\%; febrero 61.7\%). En cuanto a la variable Fase de juego, en el Mes de enero predominan las Situaciones mixtas (71.6\%), reduciéndose estas en el Mes de febrero a favor de un aumento en el trabajo de la Fase de ataque (14.9\%) y la Preparación física (23.4\%). Respecto a la variable Tipo de contenido, existe un predominio del trabajo de las Conductas técnico-tácticas colectivas de ataque (enero 76.0\%; febrero 57.4\%). Finalmente, se encuentra en la variable Línea de Juego una reducción del uso de tareas donde participa el total del equipo (enero 97.0\%; febrero 78.7\%), a favor de un trabajo más específico del portero (8.5\%) y el extremo (10.6\%) en el mes de febrero y en la variable Nivel de Oposición un aumento de las tareas Sin Oposición (enero 26.9\%; febrero 44.7\%). Además, se puede comprobar la existencia de diferencias entre las variables independientes con la variable del estudio, Mes de entrenamiento. Para establecer el grado de asociación entre variables, se muestran los Residuos Tipificados Corregidos (RTC).

Tabla $\mathrm{n}^{\circ}$ 3. Análisis descriptivo e inferencial en relación a las variables pedagógicas registradas durante los dos meses de período competitivo en la presente investigación.

\begin{tabular}{|c|c|c|c|c|c|c|c|}
\hline \multirow{2}{*}{\multicolumn{2}{|c|}{ Variables }} & \multicolumn{6}{|c|}{ Mes de entrenamiento } \\
\hline & & \multicolumn{3}{|c|}{ Mes de enero } & \multicolumn{3}{|c|}{ Mes de febrero } \\
\hline \multirow[t]{19}{*}{ SJ } & & $n$ & $\%$ & $R T C$ & $n$ & $\%$ & $R T C$ \\
\hline & $1 \times 0$ & 2 & 2.0 & -1.3 & 4 & 8.5 & 1.3 \\
\hline & $1 \times 1$ & 1 & 1.5 & -0.3 & 1 & 2.1 & 0.3 \\
\hline & $2 \times 2$ & 1 & 1.5 & 0.8 & 0 & 0.0 & -0.8 \\
\hline & $3 \times 0$ & 0 & 0.0 & -1.7 & 2 & 4.3 & 1.7 \\
\hline & $3 \times 1$ & 2 & 3.0 & -0.4 & 0 & 0.0 & 0.4 \\
\hline & $3 \times 2$ & 1 & 1.5 & 0.8 & 0 & 0.0 & -0.8 \\
\hline & $3 \times 3$ & 0 & 0.0 & $-2.1 *$ & 3 & 6.4 & $2.1 *$ \\
\hline & $4 \times 0$ & 1 & 1.5 & $-2.2 *$ & 5 & 10.6 & $2.2 *$ \\
\hline & $4 \times 2$ & 0 & 0.0 & -1.2 & 1 & 2.1 & 1.2 \\
\hline & $5 \times 1$ & 1 & 1.5 & 0.8 & 0 & 0.0 & -0.8 \\
\hline & $5 \times 2$ & 2 & 3.0 & 1.2 & 0 & 0.0 & -1.2 \\
\hline & $5 \times 3$ & 1 & 1.5 & 0.8 & 0 & 0.0 & -0.8 \\
\hline & $5 \times 5$ & 0 & 0.0 & -1.2 & 1 & 2.1 & 1.2 \\
\hline & $6 \times 6$ & 28 & 41.8 & $5.1 *$ & 0 & 0.0 & $-5.1 *$ \\
\hline & $7 \times 1$ & 1 & 1.5 & 0.8 & 0 & 0.0 & -0.8 \\
\hline & $7 \times 2$ & 1 & 1.5 & 0.8 & 0 & 0.0 & -0.8 \\
\hline & $8 \times 7$ & 2 & 3.0 & $-3.1 *$ & 10 & 21.3 & $3.1 *$ \\
\hline & $8 \times 8$ & 4 & 6.0 & 1.0 & 1 & 2.1 & -1.0 \\
\hline
\end{tabular}

Para citar este artículo utilice la siguiente referencia: Gamonales Puerto, J.M.; Gómez Carmona, C.D.; León Guzmán, K.; García Santos, D.; Gamero Portillo, M.G.; Muñoz Jiménez, J. (2019). Análisis de las tareas de entrenamiento en fútbol-base: diferencias entre dos meses durante el periodo competitivo en la categoría sub-19. SportisSci J, 5 (1), 30-52. DOI: https://doi.org/10.17979/sportis.2019.5.1.3469

http://revistas.udc.es/ 
Sportis. Revista Técnico-Científica del Deporte Escolar, Educación Física y Psicomotricidad

Sportis. Scientific Technical Journal of School Sport, Physical Education and Psychomotricity

Artículo Original. Análisis de las tareas de entrenamiento en fútbol-base: diferencias entre dos meses durante el periodo competitivo en la categoría sub-19. Vol. V, nº 1; p. 30-52, enero 2019. A Coruña. España ISSN 2386-8333

\begin{tabular}{|c|c|c|c|c|c|c|}
\hline $9 \times 1$ & 0 & 0.0 & -1.2 & 1 & 2.1 & 1.2 \\
\hline $9 \times 9$ & 3 & 4.5 & 1.5 & 0 & 0.0 & -1.5 \\
\hline $\mathrm{N} \times \mathrm{N}$ & 2 & 3.0 & $-2.0 *$ & 6 & 12.8 & $2.0 *$ \\
\hline Combinado & 14 & 20.9 & 0.0 & 10 & 21.3 & 0.0 \\
\hline POR & $n$ & $\%$ & $R T C$ & $n$ & $\%$ & $R T C$ \\
\hline Con portero & 11 & 16.4 & $-2.6 *$ & 18 & 38.3 & $2.6^{*}$ \\
\hline Sin portero & 56 & 83.6 & $2.6 *$ & 29 & 61.7 & $-2.6 *$ \\
\hline $\mathrm{FJ}$ & $n$ & $\%$ & $R T C$ & $n$ & $\%$ & $R T C$ \\
\hline Ataque & 2 & 3.0 & $-2.3^{*}$ & 7 & 14.9 & $2.3 *$ \\
\hline Defensa & 0 & 0.0 & $-2.4 *$ & 4 & 8.5 & $2.4 *$ \\
\hline Mixta & 48 & 71.6 & $2.5 *$ & 23 & 48.9 & $-2.5 *$ \\
\hline Calentamiento & 10 & 14.9 & 1.8 & 2 & 4.3 & -1.8 \\
\hline Preparación física & 7 & 10.4 & -1.9 & 11 & 23.4 & 1.9 \\
\hline CONT-G & $n$ & $\%$ & $R T C$ & $n$ & $\%$ & $R T C$ \\
\hline CTTGA & 1 & 1 & 0.8 & 0 & 0.0 & -0.8 \\
\hline CTTCA & 51 & 76 & $2.1 *$ & 27 & 57.4 & $-2.1 *$ \\
\hline GTTIA & 1 & 1 & -1.7 & 2 & 4.3 & 1.7 \\
\hline Lanzamiento & 0 & 0.0 & $-2.4 *$ & 4 & 8.5 & $2.4 *$ \\
\hline Calentamiento & 11 & 16 & 0.9 & 5 & 10.6 & -0.9 \\
\hline Preparación física & 2 & 3 & -1.7 & 5 & 10.6 & 1.7 \\
\hline Partido entrenamiento & 1 & 1 & -1.8 & 4 & 8.5 & 1.8 \\
\hline MIE & $n$ & $\%$ & $R T C$ & $n$ & $\%$ & $R T C$ \\
\hline Ejercicio de aplicación simple & 10 & 14.9 & 0.3 & 8 & 17.0 & -0.3 \\
\hline Ejercicio de aplicación compleja & 1 & 1.5 & -1.8 & 4 & 8.5 & 1.8 \\
\hline Juego simple específico & 1 & 1.5 & 0.8 & 0 & 0.0 & -0.8 \\
\hline Juego complejo inespecífico & 3 & 4.5 & 1.5 & 0 & 0.0 & -1.5 \\
\hline Juego complejo específico & 25 & 37.3 & -1.2 & 23 & 48.9 & 1.2 \\
\hline Pre-deporte / deporte adaptado & 17 & 25.4 & $2.6 *$ & 3 & 6.4 & $-2.6 *$ \\
\hline Deporte & 9 & 13.4 & 0.4 & 5 & 10.6 & -0.4 \\
\hline Competición & 1 & 1.5 & -1.8 & 4 & 8.5 & 1.8 \\
\hline $\mathrm{LJ}$ & $n$ & $\%$ & $R T C$ & $n$ & $\%$ & $R T C$ \\
\hline Portero & 0 & 0.0 & $-2.4 *$ & 4 & 8.5 & $2.4 *$ \\
\hline Lateral & 1 & 1.5 & 0.8 & 0 & 0.0 & -0.8 \\
\hline Extremo & 1 & 1.5 & $-2.2 *$ & 5 & 10.6 & $2.2 *$ \\
\hline Delantero & 0 & 0.0 & -1.2 & 1 & 2.1 & 1.2 \\
\hline Equipo & 65 & 97.0 & $3.1 *$ & 37 & 78.7 & $-3.1 *$ \\
\hline \multirow{3}{*}{$\begin{array}{l}\text { Sin oposición } \\
\text { Con oposición }\end{array}$} & $n$ & $\%$ & $R T C$ & $n$ & $\%$ & $R T C$ \\
\hline & 18 & 26.9 & $-2.0 *$ & 21 & 44.7 & $2.0 *$ \\
\hline & 49 & 73.1 & $2.0 *$ & 26 & 55.3 & $-2.0 *$ \\
\hline
\end{tabular}

Nota. SJ: Situación de Juego; NxN: Agrupación de jugadores superior a 9x9; POR: Presencia de portero; FJ: Fase de Juego; CONT-G: Tipo de contenido; CTTGA: Contenido técnico-táctico grupal de ataque; CTTCA: Contenido técnicotáctico colectivo de ataque; GTTIA: Contenido técnico-táctico individual de ataque; MIE: Medio de Iniciación al Entrenamiento; LJ: Línea de Juego; NO: Nivel de Oposición; * RTC $=|1.96|$

Los resultados de las diferencias entre las variables planteadas en el estudio de caracterización de los meses competitivos en relación a la variable Mes de entrenamiento, se muestran en la tabla 4. En el estudio, se observa un predominio de asociación moderado en las variables analizadas, excepto en la variable Situación de Juego con un grado de asociación alto y en las variables Presencia de Portero y Nivel de Oposición con un grado de asociación bajo según Crewson (2006). 
Artículo Original. Análisis de las tareas de entrenamiento en fútbol-base: diferencias entre dos meses durante el periodo competitivo en la categoría sub-19. Vol. V, no. 1; p. 30-52, enero 2019. A Coruña. España ISSN 2386-8333

Tabla $\mathrm{n}^{\circ}$ 4. Relación entre la variable Mes de entrenamiento y las variables independientes del estudio.

\begin{tabular}{cccccccc}
\hline Variables & \multicolumn{6}{c}{ Mes de entrenamiento } \\
& $\chi 2$ & $g l$. & Sig. & $\varphi c$ & Sig. & Nivel de asociación \\
\hline Situaciones de juego (SJ) & 58.423 & 21 & $\mathbf{0 . 0 0 0}$ & $*$ & 0.716 & 0.000 & Alto \\
Presencia de portero (POR) & 6.972 & 1 & $\mathbf{0 . 0 0 8}$ & $*$ & 0.247 & 0.008 & Bajo \\
Fase de juego (FJ) & 18.875 & 4 & $\mathbf{0 . 0 0 1}$ & $*$ & 0.407 & 0.001 & Moderado \\
Tipo de contenido (CONT-G) & 17.758 & 7 & $\mathbf{0 . 0 1 3}$ & $*$ & 0.395 & 0.013 & Moderado \\
Medio de iniciación al entrenamiento (MIE) & 15.827 & 7 & $\mathbf{0 . 0 2 7}$ & $*$ & 0.373 & 0,027 & Moderado \\
Línea de juego / grupo de jugadores (LJ) & 13.252 & 4 & $\mathbf{0 . 0 1 0}$ & $*$ & 0.341 & 0.010 & Moderado \\
Nivel de oposición (NO) & 3.895 & 1 & $\mathbf{0 . 0 4 8}$ & $*$ & 0.185 & 0.048 & Bajo \\
\hline
\end{tabular}

$* p<0,05 ;$ Nivel de asociación según Crewson (2006).

\section{Discusión}

Este trabajo tiene como objetivo caracterizar las tareas de entrenamiento diseñadas y desarrolladas por un único entrenador de fútbol-base, en la categoría juvenil, durante dos meses competitivos, además de comparar las características pedagógicas de las tareas entre ambos periodos de entrenamiento. Los principales resultados obtenidos indican que el técnico especialista en fútbol utilizó una gran variedad de variables pedagógicas en sus sesiones para el trabajo de las capacidades físico-técnico-tácticas de los jugadores de fútbol en categoría juvenil a través de la utilización de juegos reducidos (JR), en situaciones de 6x6, 8x7 y combinadas sin portero, donde todo el equipo trabajaba conjuntamente, en fase de juego mixta (transiciones ataque-defensa) y en mayor medida el contenido de conductas técnicotácticas colectivas de ataque. En relación al segundo objetivo, las principales diferencias existentes en el diseño de tareas entre los meses analizados están relacionadas fuertemente con las variables pedagógicas propuestas en el estudio analizadas a través de la herramienta SIATE.

En la literatura científica, existen estudios relacionados con las variables pedagógicas en otras modalidades deportivas, concretamente con las SJ (Cañadas et al., 2013), los CONT$G$ (Cañadas e Ibáñez, 2010; Cañadas et al., 2015), los MIE (Cañadas et al., 2011; Cañadas et al., 2009), las $F J$ (Cañadas et al., 2012), y la relación entre las variables pedagógicas del entrenamiento (Cañadas et al., 2013). En fútbol, únicamente existe un trabajo previo que analice las tareas de entrenamiento en categorías base con el objetivo de identificar la asociación entre los medios de iniciación al entrenamiento y las diferentes variables pedagógicas que las definen (Gómez-Carmona et al., 2018), encontrando una alta asociación.

\footnotetext{
Para citar este artículo utilice la siguiente referencia: Gamonales Puerto, J.M.; Gómez Carmona, C.D.; León Guzmán, K.; García Santos, D.; Gamero Portillo, M.G.; Muñoz Jiménez, J. (2019). Análisis de las tareas de entrenamiento en fútbol-base: diferencias entre dos meses durante el periodo competitivo en la categoría sub-19. SportisSci J, 5 (1), 30-52. DOI: https://doi.org/10.17979/sportis.2019.5.1.3469

http://revistas.udc.es/
} 


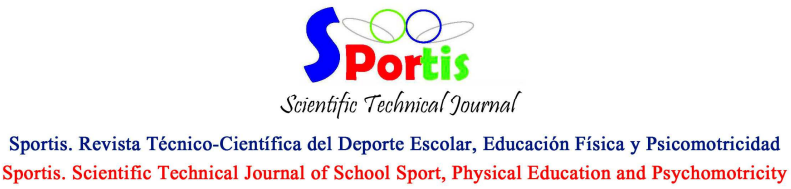

Sportis. Scientific Technical Journal of School Sport, Physical Education and Psychomotricity

Artículo Original. Análisis de las tareas de entrenamiento en fútbol-base: diferencias entre dos meses durante el periodo competitivo en la categoría sub-19. Vol. V, no $.1 ;$ p. 30-52, enero 2019. A Coruña. España ISSN 2386-8333

En relación a la variable $S J$ en el periodo competitivo analizado predominan los JR de

$6 \mathrm{x} 6,8 \times 7$ y combinados. Estas tareas de entrenamiento poseen bastantes exigencias cinemáticas y fisiológicas semejantes al juego real (Casamichana et al., 2012), y que mejoran la toma de decisiones (Davids et al., 2013). Por tanto, las situaciones reducidas están próximas a las demandas que exige la competición, y, además, permite a los jugadores construir su propio aprendizaje (Gil et al., 2016). En cambio, diversas investigaciones afirman que existe un déficit en el desarrollo de las capacidades físicas a través de los JR debido a que no se alcanzan las demandas de carrera a alta intensidad (Nevado-Garrosa y Suarez-Arrones, 2015; San Román-Quintana, Casamichana, Castellano y Calleja, 2014). Por tanto, el uso de situaciones reducidas de juego durante los entrenamientos debe ser muy bien planificado, debido a que la variabilidad de jugadores, así como a las dimensiones del espacio, pues serán determinantes en las capacidades físicas, técnicas, tácticas o psicológicas a trabajar, siempre que se reproduzcan las mismas condiciones a las que el jugador se enfrentará durante la competición.

Por el contrario, en relación a la variable $P O R$, se encuentra una gran ausencia en las tareas de entrenamiento. Esta situación en los juegos de toma de decisiones puede alterar la lógica interna del fútbol (Parlebas, 2001). Por lo cual, como consecuencia del carácter cambiante de los deportes colectivos de colaboración-oposición (Ortega-Toro y Sainz de Baranda, 2003), se recomienda realizar juego con la presencia de porteros, para aumentar la toma de decisiones y las exigencias físicas, así como la disposición táctica de los jugadores (Travassos, Gonçalves, Marcelino, Monteiro y Sampaio, 2014). Por tanto, el trabajo conjunto del portero con el equipo durante las tareas diseñadas en las sesiones de entrenamiento es muy importante para desarrollar situaciones técnico-tácticas similares a las producidas durante la competición.

Con respecto a la $F J$ y $C O N T-G$, el técnico deportivo especialista en fútbol diseña en los meses analizados, tareas de entrenamiento mixtas donde predominan los contenidos CTTCA como consecuencias del contexto deportivo. Por ello, para diseñar las tareas de entrenamiento en el fútbol-base, se debe partir del patrón principal que es la realidad de la competición (Casamichana et al., 2012; Hill-Haas et al., 2011) y la idoneidad del espacio para desarrollar los entrenamientos (Fradua et al., 2012).

\footnotetext{
Para citar este artículo utilice la siguiente referencia: Gamonales Puerto, J.M.; Gómez Carmona, C.D.; León Guzmán, K.; García Santos, D.; Gamero Portillo, M.G.; Muñoz Jiménez, J. (2019). Análisis de las tareas de entrenamiento en fútbol-base: diferencias entre dos meses durante el periodo competitivo en la categoría sub-19. SportisSci J, 5 (1), 30-52. DOI: https://doi.org/10.17979/sportis.2019.5.1.3469

http://revistas.udc.es/
} 


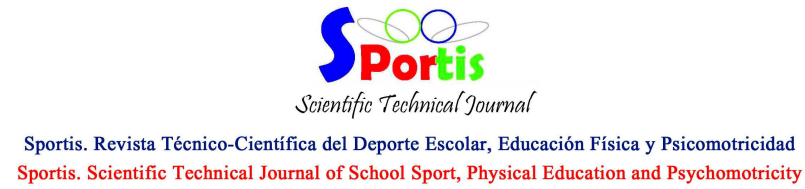

Sportis. Scientific Technical Journal of School Sport, Physical Education and Psychomotricity

Artículo Original. Análisis de las tareas de entrenamiento en fútbol-base: diferencias entre dos meses durante el periodo competitivo en la categoría sub-19. Vol. V, no $.1 ;$ p. 30-52, enero 2019. A Coruña. España ISSN 2386-8333

En relación a los $M I E, L J$ y $N O$, el entrenador de fútbol-base diseña juegos complejos específicos de equipo y con oposición. Estos resultados son muy similares a los estudios existentes en el fútbol convencional (Casamichana et al., 2012; Ortega-Toro y Sainz de Baranda, 2003; Fradua et al., 2012). Sin embargo, son totalmente diferentes a los estudios existentes con SIATE (Cañadas et al., 2012), debido a que están centrados principalmente en baloncesto y el contexto deportivo es totalmente diferente al fútbol. Por tanto, es muy importante la adaptación de las tareas a la lógica interna de la modalidad deportiva a trabajar para una mejora del aprendizaje del jugador de fútbol.

Por tanto, en resumen, a través del registro y posterior análisis de las tareas de entrenamiento durante el periodo competitivo se podrá extraer información básica e importante para el diseño de las tareas de entrenamiento para los técnicos deportivos en la especialidad de fútbol, siendo más específico el trabajo de las variables pedagógicas para un mejor desarrollo físico-técnico-táctico del jugador adaptado a las exigencias de la competición. Para ello, SIATE es una herramienta óptima para caracterizar, organizar y estructurar las tareas de entrenamiento cuando no se dispone de grandes recursos materiales y tecnológicos (Gómez-Carmona et al., 2018; Ibáñez et al. 2016).

En relación al segundo objetivo, el análisis inferencial confirma que la variable Mes de entrenamiento estaba relacionada con las variables planteadas para el estudio, concretamente $S J, P O R, F J, C O N T-G, M I E, L J$ y $N O$ que determinan la existencia de diferencias entre los dos meses analizados durante el periodo competitivo.

Las diferencias existentes entre las $S J$ y el Mes de entrenamiento, muestra que el entrenador diseña tareas de $6 x 6$ para el Mes de enero, y con respecto al Mes de febrero, predominan las situaciones reducidas de $8 \times 7$ y $3 \times 3$. Estos JR son situaciones específicas del fútbol, y están en consonancia con los nuevos métodos de planificación (Leo-Marcos et al., 2012) con la finalidad de conseguir jugadores con un gran bagaje técnico-táctico. Por tanto, el entrenador analizado se aleja de las tareas cerradas y analíticas orientadas a la repetición y reproducción sistemática.

Con respecto a la $P O R$, los resultados muestran que hay diferencias entre los meses de entrenamientos analizados. Existe una mayor probabilidad de lo esperado de que las tareas de

\footnotetext{
Para citar este artículo utilice la siguiente referencia: Gamonales Puerto, J.M.; Gómez Carmona, C.D.; León Guzmán, K.; García Santos, D.; Gamero Portillo, M.G.; Muñoz Jiménez, J. (2019). Análisis de las tareas de entrenamiento en fútbol-base: diferencias entre dos meses durante el periodo competitivo en la categoría sub-19. SportisSci J, 5 (1), 30-52. DOI: https://doi.org/10.17979/sportis.2019.5.1.3469

http://revistas.udc.es/
} 


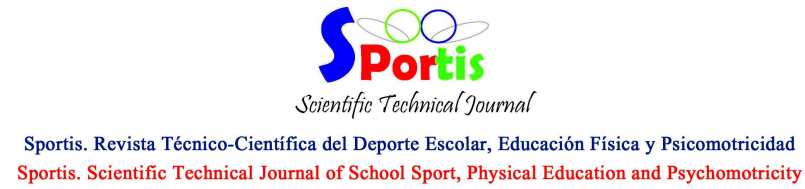

Sportis. Scientific Technical Journal of School Sport, Physical Education and Psychomotricity

Artículo Original. Análisis de las tareas de entrenamiento en fútbol-base: diferencias entre dos meses durante el periodo competitivo en la categoría sub-19. Vol. V, no . 1; p. 30-52, enero 2019. A Coruña. España ISSN 2386-8333

entrenamiento diseñadas por el entrenador en el Mes de febrero sean con portero. Por tanto, son juegos que aumenta la toma de decisiones de los jugadores con respecto a las tareas sin portero. El Mes de enero, se alejaría de la propuesta de diseño de tarea de entrenamiento de Ortega-Toro y Sainz de Baranda (2003), donde predominan las actividades en espacios reducidos con portero. Por ello, se recomienda realizar tareas de entrenamiento globales e integradas, con la finalidad de mejorar la enseñanza en el fútbol base. De esta manera, se conseguirá jugadores con una mayor riqueza técnico-táctica y capaces de resolver de forma muy diferentes los distintos problemas a los que se enfrentan en el terreno de juego.

La relación entre la $F J$ y el Mes de entrenamiento, muestra que existen diferencias entre los mesociclos analizados. En el Mes de enero, predominan las tareas mixtas. Sin embargo, en el Mes de febrero, las tareas diseñadas por el técnico deportivo, especialista en fútbol, son de ataque y/o defensa. Es decir, son acciones enfocadas a trabajar o mejorar determinados aspectos de forma específica en el fútbol. Esto puede deberse a los resultados o errores cometidos por los jugadores durante el partido. Estos resultados no coinciden con la literatura existente (Cañadas et al., 2012). Por consiguiente, el entrenador diseñó tareas centradas en trabajar transiciones de ataque o defensa concretas, en lugar de hacerlo de forma mixta, con la finalidad de que los jugadores estén en óptimas condiciones para el desarrollo de la competición (Stølen, Chamari, Castagna y Wisløff, 2005). Por tanto, los entrenadores en el fútbol base tienen que diseñar tareas de entrenamiento mixtas, con la finalidad de comprender el momento en el que se pasa de ataque a defensa y defensa al ataque, una vez que se produce la pérdida o recuperación del balón.

Por otro lado, la relación entre la $C O N T-G$, y el Mes de entrenamiento, muestra que existen diferencias entre los mesociclos analizados. En el Mes de enero, predominan las tareas CTTCA y en el Mes de febrero, el tipo de contenido más utilizado es el lanzamiento a portería. Estos datos son totalmente diferentes a los estudios existentes con SIATE (Cañadas et al., 2012; Cañadas et al., 2015) e incluso no corroboran con los datos analizados previamente en la literatura en fútbol. Sin embargo, tienen cierta similitud con el trabajo de Gómez-Carmona et al., (2018). El entrenador desarrolla principalmente juegos complejos específico de 6x6, sin porteros, mixtos y se trabajan CTTCA con oposición. Por tanto, puede deberse a la escasa muestra analizada, tanto en el trabajo de Gómez-Carmona et al., (2018),

\footnotetext{
Para citar este artículo utilice la siguiente referencia: Gamonales Puerto, J.M.; Gómez Carmona, C.D.; León Guzmán, K.; García Santos, D.; Gamero Portillo, M.G.; Muñoz Jiménez, J. (2019). Análisis de las tareas de entrenamiento en fútbol-base: diferencias entre dos meses durante el periodo competitivo en la categoría sub-19. SportisSci J, 5 (1), 30-52. DOI: https://doi.org/10.17979/sportis.2019.5.1.3469
}

http://revistas.udc.es/ 


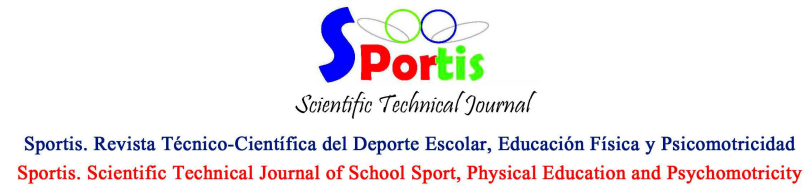

Sportis. Scientific Technical Journal of School Sport, Physical Education and Psychomotricity

Artículo Original. Análisis de las tareas de entrenamiento en fútbol-base: diferencias entre dos meses durante el periodo competitivo en la categoría sub-19. Vol. V, no $.1 ;$ p. 30-52, enero 2019. A Coruña. España ISSN 2386-8333

así como en el presente estudio. Lo recomendable sería analizar como mínimo una temporada, y comprobar si los resultados están próximos a los citados por Cañadas e Ibáñez (2016), donde las tareas deben buscar el desarrollo de conductas táctico-técnicas, así como favorecer el desarrollo cognitivo de los jugadores (Gómez-Carmona et al., 2018).

Las diferencias existentes entre los MIE y el Mes de entrenamiento, muestra que el entrenador diseña tareas de predeporte / deporte adaptado para el Mes de enero. Con respecto al Mes de febrero, no predominan las tareas de predeporte / deporte adaptado. El predeporte / deporte adaptado son situaciones de juego dentro del contexto de juego real (Cañadas e Ibáñez, 2016) con modificaciones de los elementos claves. Es decir, la toma de decisiones de los jugadores en el predeporte / deporte adaptado dependerá de la modificación del espacio (Fradua et al., 2012) o el reglamento, las acciones técnica-tácticas, la comunicación, el tiempo y la estrategia (Ortega-Toro y Sainz de Baranda, 2003). Por tanto, los jugadores adquieren mayor riqueza de aprendizaje significativo en el Mes de enero, pues tienen que tomar un mayor número de decisiones, con la finalidad de entender el juego. Para ello, será necesario que los entrenadores diseñen y apliquen tareas de entrenamiento con una correcta metodología que permita crear jugadores más inteligentes y capaces de leer las situaciones de juego correctamente.

Con respecto a la $L J$, los resultados muestran que hay diferencias entre los meses de entrenamiento analizados. En el Mes de enero, predominan las situaciones reducidas de equipo. Por el contrario, en el Mes de febrero, existe una mayor probabilidad de que las tareas sean centradas para trabajas los puestos específicos de portero y extremo. No existen estudios relacionados con los resultados. Por último, la relación existente entre NO y el Tipo de Competición, los resultados muestran que en el Mes de enero, el entrenador diseña tareas con oposición, y por el contrario en el Mes de febrero, sin oposición. Por tanto, en el fútbol base se tienen que diseñar tareas de entrenamiento próximas al juego real para estar cercano a lo que citan Casamichana et al., (2012) o Hill-Haas, et al., (2011). Además, deben de predominar las situaciones de juego que fomenten la toma de decisiones. Por ello, los entrenadores deben saber que la toma de decisiones se entrena, y se tienen que diseñar tareas de entrenamiento para conseguir jugadores más completos y para que sepan a qué juegan, con la finalidad de que entiendan el juego.

\footnotetext{
Para citar este artículo utilice la siguiente referencia: Gamonales Puerto, J.M.; Gómez Carmona, C.D.; León Guzmán, K.; García Santos, D.; Gamero Portillo, M.G.; Muñoz Jiménez, J. (2019). Análisis de las tareas de entrenamiento en fútbol-base: diferencias entre dos meses durante el periodo competitivo en la categoría sub-19. SportisSci J, 5 (1), 30-52. DOI: https://doi.org/10.17979/sportis.2019.5.1.3469

http://revistas.udc.es/
} 


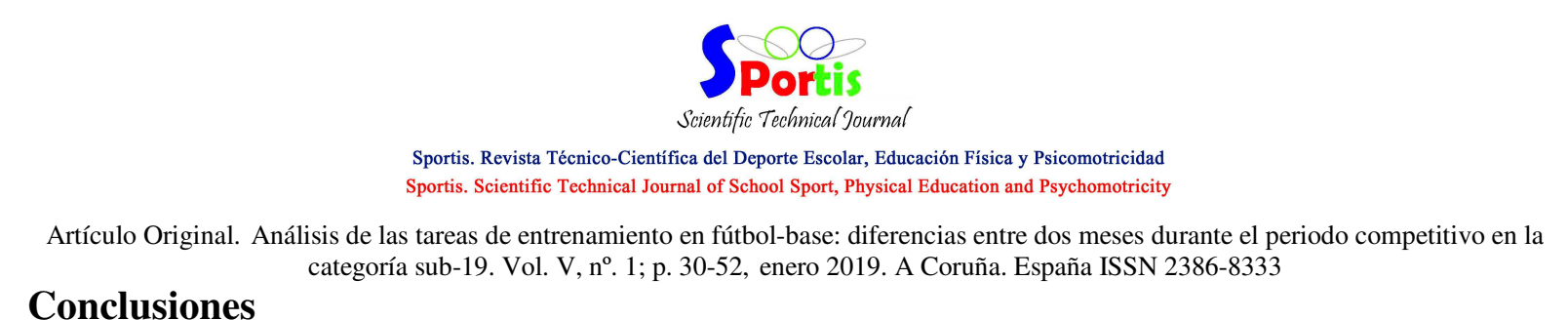

\section{Conclusiones}

El entrenador de fútbol-base en esta investigación utiliza predominantemente para el desarrollo de las capacidades físicas, técnicas y tácticas del jugador de fútbol en categoría juvenil las siguientes variables pedagógicas: (a) Medios de Iniciación al entrenamiento: Juego complejo específico y Deporte adaptado; (b) Situaciones de Juego: 6x6 y 8x7; (c) Presencia de Portero: Sin presencia de portero; (d) Fase de Juego: Mixta, combinación de las fases de ataque y defensa dentro de la misma tarea; (e) Tipo de Contenido: Conductas técnico-tácticas colectivas de ataque (CTTCA); (f) Línea de Juego: Equipo, todos los puestos trabajan de forma conjunta, y; (g) Nivel de Oposición: Con oposición.

En la comparativa de diseño de las tareas de entrenamiento en relación a las variables pedagógicas se encuentran diferencias significativas en las variables de estudio analizadas (SJ, POR, FJ, CONT-G, MIE, LJ y NO), encontrando un grado de asociación principalmente moderado. La herramienta SIATE, gracias a la formación previa de los observadores, ha demostrado ser una herramienta útil para el registro y posterior análisis de las variables pedagógicas de las tareas de entrenamiento diseñadas y llevadas a la práctica durante las sesiones de entrenamiento.

En lo que respecta a la aplicación práctica, un conocimiento más amplio sobre las características de las tareas realizadas durante las sesiones de entrenamiento en cuanto a las variables pedagógicas trabajadas va a permitir determinar el perfil de dichas acciones de trabajo, y a partir de su análisis, poder mejorar su diseño, así como la aplicación de las mismas durante las sesiones de entrenamiento con la finalidad de crear jugadores de fútbol base más inteligentes capaces de resolver las diferentes situaciones que se dan en el juego.

Aunque la investigación realizada recoge dos meses completos de entrenamiento de un equipo federado juvenil de categoría regional, este trabajo presenta diferentes limitaciones entre las que destacan tener como muestra a un sólo equipo de dieciocho jugadores, donde un único entrenador con unas características propias de experiencia y formación fue el encargado de diseñar y llevar a la práctica las tareas durante las sesiones de entrenamiento. Por tanto, para investigaciones futuras se debería profundizar en los objetivos de esta investigación realizándose la misma indagación con un mayor número de jugadores, así como analizar un 


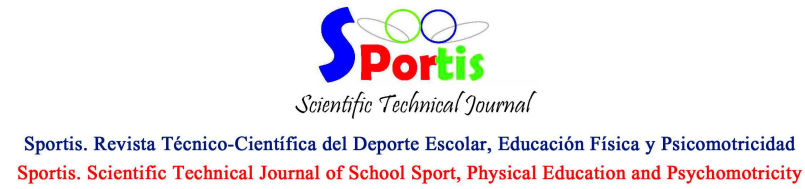

Artículo Original. Análisis de las tareas de entrenamiento en fútbol-base: diferencias entre dos meses durante el periodo competitivo en la categoría sub-19. Vol. V, no . 1; p. 30-52, enero 2019. A Coruña. España ISSN 2386-8333

mayor número de categorías, y un mayor número de entrenamientos. Además, sería interesante realizar el análisis con diferentes equipos y entrenadores con diferente experiencia y formación. De esta manera, se podría analizar los tipos de metodologías utilizados por los entrenadores en el fútbol-base.

\section{Referencias bibliográficas}

1. Alarcón, F., Cárdenas, D., Miranda, M.T., Ureña, N., y Piñar, M.I. (2010). La metodología de enseñanza en los deportes de equipo. Revista de investigación en educación, 7, 91-103.

2. Arjol, J.L. (2012). La planificación actual del entrenamiento en fútbol: análisis comparado del enfoque estructurado y la periodización táctica. Acción Motriz, 8, 2728.

3. Ato, M., López, J.J., y Benavente, A. (2013). Un sistema de clasificación de los diseños de investigación en psicología. Anales de Psicología, 29(3), 1038-1059. DOI: http://dx.doi.org/10.6018/analesps.29.3.178511

4. Barraza, A. (2007). Apuntes sobre metodología de la investigación: confiabilidad. Revista INED, 2 (6), 6-10.

5. Bompa, T. (1993). Theory and methodology of Training the to athletics Performance. Publishing Company. Lowa/Hunt.

6. Cañadas, M., e Ibáñez, S.J. (2010). La planificación de los contenidos de entrenamiento de baloncesto con equipos de iniciación. E-Balonmano: Revista de Ciencias del Deporte, 6(1), 49-65.

7. Cañadas, M., Ibáñez, S.J., Feu, S., García-Rubio, J., y Parejo, I. (2011). Análisis de los medios de entrenamiento en un equipo minibasket y la influencia de un programa formativo para el entrenador. Un estudio de caso. ÁGORA para la Educación Física y el Deporte, 13(3), 363-382.

8. Cañadas, M., Ibáñez, S.J., García-Rubio, J., Parejo, I., y Feu, S. (2012). Estudio de las fases de juego a través del análisis del entrenamiento deportivo en categoría minibasket. Cuadernos de Psicología del Deporte, 12(2), 73-82.

\footnotetext{
Para citar este artículo utilice la siguiente referencia: Gamonales Puerto, J.M.; Gómez Carmona, C.D.; León Guzmán, K.; García Santos, D.; Gamero Portillo, M.G.; Muñoz Jiménez, J. (2019). Análisis de las tareas de entrenamiento en fútbol-base: diferencias entre dos meses durante el periodo competitivo en la categoría sub-19. SportisSci J, 5 (1), 30-52. DOI: https://doi.org/10.17979/sportis.2019.5.1.3469

http://revistas.udc.es/
} 


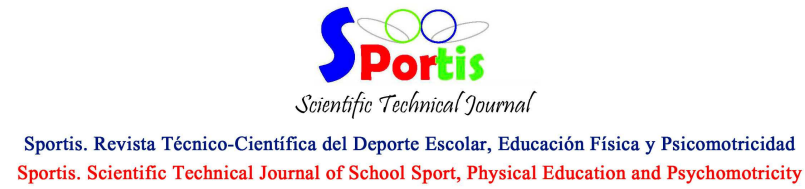

Sportis. Scientific Technical Journal of School Sport, Physical Education and Psychomotricity

Artículo Original. Análisis de las tareas de entrenamiento en fútbol-base: diferencias entre dos meses durante el periodo competitivo en la categoría sub-19. Vol. V, no . 1; p. 30-52, enero 2019. A Coruña. España ISSN 2386-8333

9. Cañadas, M., Ibáñez, S.J., García-Rubio, J., Parejo, I., y Feu, S. (2013). Game situations in youth basketball practices. Revista Internacional de Medicina y Ciencias de la Actividad Física y del Deporte, 13(49), 41-54.

10. Cañadas, M., Ibáñez, S.J., y Leite, N. (2015). A novice coach's planning of the technical and tactical content of youth basketball training: A case study. International Journal of Performance Analysis in Sport, 15(2), 572-587. DOI: http://doi.org/10.1080/24748668.2015.11868815

11. Cañadas, M., Parejo, I., Ibáñez, S.J. García-Rubio, J., y Feu, S. (2009). Relación entre las variables pedagógicas de los entrenadores de baloncesto que trabajan en etapas de formación. Habilidades motrices, Revista de Ciencias de la Actividad Física y del Deporte, 32, 44-52.

12. Cañadas, M., Rodríguez, G., Feu, S., Parejo, I., y García-Rubio, J. (2013). Relathionship between pedagogical content knowledge and coaching methods. Revista de Psicología del Deporte, 22(1), 183-186.

13. Casamichana, D., Castellano, J., y Castagna, C. (2012). Comparing the physical demands of friendly matches and small-sided games in semiprofessional soccer players. The Journal of Strength \& Conditioning Research,26(3), 837-843. DOI: http://doi.org/10.1519/JSC.0b013e31822a61cf

14. Castejón, F.J. (2015). La investigación en iniciación deportiva válida para el profesorado de educación física en ejercicio. Retos: Nueva tendencias en educación física, deporte y recreación, 28, 263-269.

15. Clemente, F.M., Martins, F.M., y Mendes, R.S. (2015). How coaches use Their knowledge to develop small-side soccer games: a case study. South African Journal for Research in Sport Physical Education and Recreation, 37(1), 1-11.

16. Crewson, P (2006). Applied statistics handbook. AcaStat Software, Leesburg.

17. Davids, K., Araújo, D., Correia, V., y Vilar, L. (2013). How small-sided and conditioned games enhance acquisition of movement and decision-making skills. Exercise and sport sciences reviews,4l(3), 154-161. DOI: http://doi.org/10.1097/JES.0b013e318292f3ec

18. Field, A. (2009). Discovering statistics using SPSS $\left(3^{a}\right.$ ed.). London: Sage Publications Ltd. 


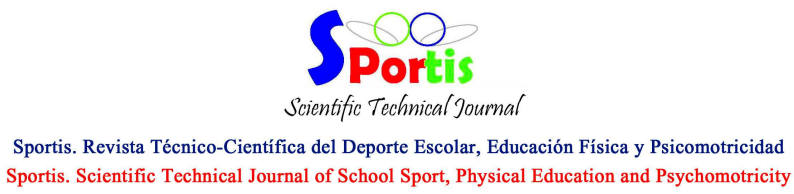

Sportis. Scientific Technical Journal of School Sport, Physical Education and Psychomotricity

Artículo Original. Análisis de las tareas de entrenamiento en fútbol-base: diferencias entre dos meses durante el periodo competitivo en la categoría sub-19. Vol. V, no $.1 ;$ p. 30-52, enero 2019. A Coruña. España ISSN 2386-8333

19. Fradua, L., Zubillaga, A., Caro, O., Fernández-García, A., Ruiz-Ruiz, C., y Tenga, A. (2012). Designing small-sided games for training tactical aspects in soccer: extrapolating pitch sizes from full-size profesional matches. Journal of Sports Sciences, 31(6), 573-581. DOI: http://doi.org/10.1080/02640414.2012.746722

20. Gamonales, J.M., Muñoz, J., León, K., e Ibáñez, S.J. (2018). Entrenamiento y confiabilidad entre observadores para el análisis del fútbol a 5 para personas ciegas. Retos, Nuevas tendencias en Educación Física, Deporte y Recreación, 34, 155-161.

21. García-Angulo, A., y García-Angulo, F.J. (2018). Percepción de la dificultad de tareas analíticas frente a globales en jóvenes jugadores de fútbol. Sportis: Revista TécnicoCientífica del Deporte Escolar, Educación Física y Psicomotricidad, 4(2), 306-330. DOI: http://doi.org/10.17979/sportis.2018.4.2.3344

22. García, J.A., y Ruiz, L.M. (2003). Análisis comparativo de dos modelos de intervención en el aprendizaje del balonmano. Revista de Psicología del Deporte, 12(1), 55-66.

23. Gil, A., Moreno, P., Claver, F., Moreno, A., y Del Villar, F. (2016). Manipulación de los condicionantes de la tarea en Educación Física: Una propuesta desde la pedagogía no lineal. Retos: Nueva tendencias en educación física, deporte y recreación, 29, 2227.

24. Gómez-Carmona, C. D., García-Rubio, J., Muñoz, J., \& Gamonales, J. M. (2018). Relación entre el medio de iniciación al entrenamiento y las variables pedagógicas que definen las tareas en el fútbol. TRANCES. Transmisión del Conocimiento Educativo y de la Salud, 10(1), 401-420.

25. González Campos, G. (2016). Estilos de enseñanza de entrenadores y su relación con la ansiedad de los jugadores en diferentes categorías de fútbol base. Sportis: Revista Técnico-Científica del Deporte Escolar, Educación Física y Psicomotricidad,2(3), 390-411. DOI: http://doi.org/10.17979/sportis.2016.2.3.1721

26. González-Espinosa, S., Ibáñez, S.J., Feu, S., y Galatti, L.R. (2017). Programas de intervención para la enseñanza deportiva en el contexto escolar, PETB y PEAB. Retos: Nueva tendencias en educación física, deporte y recreación, 31, 103-106.

\footnotetext{
Para citar este artículo utilice la siguiente referencia: Gamonales Puerto, J.M.; Gómez Carmona, C.D.; León Guzmán, K.; García Santos, D.; Gamero Portillo, M.G.; Muñoz Jiménez, J. (2019). Análisis de las tareas de entrenamiento en fútbol-base: diferencias entre dos meses durante el periodo competitivo en la categoría sub-19. SportisSci J, 5 (1), 30-52. DOI: https://doi.org/10.17979/sportis.2019.5.1.3469 http://revistas.udc.es/
} 


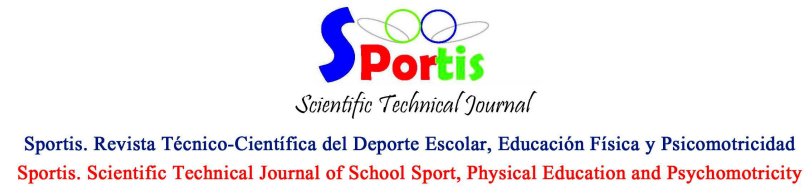

Sportis. Scientific Technical Journal of School Sport, Physical Education and Psychomotricity

Artículo Original. Análisis de las tareas de entrenamiento en fútbol-base: diferencias entre dos meses durante el periodo competitivo en la categoría sub-19. Vol. V, no $.1 ;$ p. 30-52, enero 2019. A Coruña. España ISSN 2386-8333

27. Gracia, F., García-Rubio, J., Cañadas, M., e Ibáñez, S.J. (2014). Heart rate differences in small sided games in formativa basketball. Journal of Sports Sciences, 10(1), 2330.

28. Gray, S., y Sproule, J. (2011). Developing pupils' performance in team invasion games. Physical Education and Sport Pedagogy, 16(1), 15-32.

29. Hernández, E., Ortega, E., y Palao, J.M. (2016). Efecto de distintos planteamientos metodológicos en clases de educación física sobre el aprendizaje de tres habilidades de voleibol. SPORT TK-Revista EuroAmericana de Ciencias del Deporte, 5(2), 59-68.

30. Hill-Haas, S.V., Dawson, B.T., Impellizzeri, F.M., y Coutts, A.J. (2011). Physiology of small-sided games training in soccer: a systematic review. Sports Medicine, 41, 199-220. DOI:

31. http://doi.org/10.2165/11539740-000000000-00000

32. Ibáñez, S.J., Feu, S., y Cañadas, M. (2016). Sistema integral para el análisis de las tareas de entrenamiento, SIATE, en deportes de invasión. E-balonmano.com: Revista de Ciencias del Deporte, 12(1), 3-30.

33. Jones, R.L., Armour, K., y Potrac, P. (2002). Understanding the Coaching process: a framework for social analysis. Quest, 54(1), 34-48. DOI: http://doi.org/10.1080/00336297.2002.10491765

34. Leo-Marcos, F.M., Pulido-González, J.J., Sánchez-Oliva, D., Candela, J.M., y GarcíaCalvo, T. (2012). Entrenamiento de las capacidades condicionales a través de un microciclo de competición basado en el modelo de juego en un equipo de fútbol. Acción Motriz, 10, 84-102.

35. Newell, J., Aitchison, T., y Grant, S. (2014). Statistics for sports and exercise science: a practical approach. Routledge.

36. Ortega-Toro, E., y Sainz de Baranda, P. (2003). El diseño de tareas en el fútbol base: su aplicabilidad al puesto específico del portero. Cuadernos de psicología del deporte, $3(1), 15-32$.

37. Parlebas, P. (2001). Juegos, deportes y sociedades. Léxico de praxiología motriz. Barcelona: Paidotribo.

\footnotetext{
Para citar este artículo utilice la siguiente referencia: Gamonales Puerto, J.M.; Gómez Carmona, C.D.; León Guzmán, K.; García Santos, D.; Gamero Portillo, M.G.; Muñoz Jiménez, J. (2019). Análisis de las tareas de entrenamiento en fútbol-base: diferencias entre dos meses durante el periodo competitivo en la categoría sub-19. SportisSci J, 5 (1), 30-52. DOI: https://doi.org/10.17979/sportis.2019.5.1.3469

http://revistas.udc.es/
} 


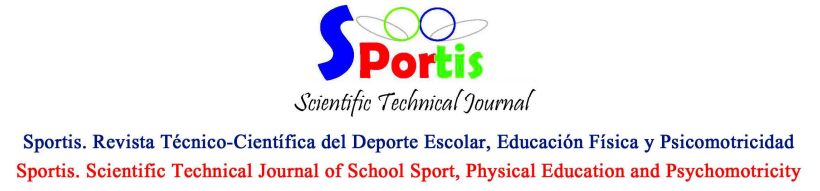

Artículo Original. Análisis de las tareas de entrenamiento en fútbol-base: diferencias entre dos meses durante el periodo competitivo en la categoría sub-19. Vol. V, no $.1 ;$ p. 30-52, enero 2019. A Coruña. España ISSN 2386-8333

38. Randolph, J.J. (2005). Free-Marginal Multirater Kappa (multirater Kfree): An Alternative to Fleiss' Fixed-Marginal Multirater Kappa. Joensuu Learning and Instruction Symposium 2005, University of the Joensuu, Finland.

39. Sáenz-López, P., Feu, S., e Ibáñez, S.J. (2006). Estudio de la participación de los jugadores españoles de baloncesto en las distintas categorías de la selección nacional. Apunts, Educación Física y Deporte, 85, 36-45.

40. Sáenz-López, P., Ibáñez, S.J., Giménez, J., Sierra, A., y Sánchez, M. (2005). Multifactor characteristics in the process of Development of the male expert basketball player in Spain. International Journal of Sport Psychology, 36(2), 151-171.

41. Sáenz-López, P., Jiménez, A.C., Giménez J., e Ibáñez, S.J. (2007). La autopercepción de las jugadores de baloncesto expertas respecto a sus procesos de formación. Cultura, Ciencia y Deporte, 7(3), 35-41. DOI: http://dx.doi.org/10.12800/ccd.v3i7.156

42. Sánchez-Sánchez, J., Yagüe, J.M., Fernández, R.C., y Petisco, C. (2014). Efectos de un entrenamiento con juegos reducidos sobre la técnica y la condición física de jóvenes futbolistas. RICYDE: Revista Internacional de Ciencias del Deporte, 10(37), 221-234. DOI: http://dx.doi.org/10.5232/ricyde2014.03704

43. Sarmento, H., Anguera, M.T., Campaniço, J., y Leitão, J. (2010). Development and validation of a notacional system to study the offensive process in football. Medicina (Kaunas), 46(6), 401-407. DOI: http://dx.doi.org/10.3390/medicina46060056

44. Stølen, T., Chamari, K., Castagna, C., y Wisløff, U. (2005). Physiology of soccer. Sports medicine, 35(6), 501-536.

45. Thorpe, R., Bunker, D., y Almond, I. (1986). Rethinking games teaching. Department of Physical Education and Sports Science, University of Technology.

46. Torres-Ronda, L., Ric, A., Llabres-Torres, I., De las Heras, B., y Del Alcazar, X.S. (2016). Position-dependent cardiovascular response and time-motion analysis during training drills and friendly matches in elite male basketball player. The Journal of Strength \& Conditioning Research, 30(1), 60-70. DOI: http://doi.org/10.1519/JSC.0000000000001043

47. Travassos, B., Gonçalves, B., Marcelino, R., Monteiro, R., y Sampaio, J. (2014). How perceiving additional targets modifies teams' tactical behavior during football small-

\footnotetext{
Para citar este artículo utilice la siguiente referencia: Gamonales Puerto, J.M.; Gómez Carmona, C.D.; León Guzmán, K.; García Santos, D.; Gamero Portillo, M.G.; Muñoz Jiménez, J. (2019). Análisis de las tareas de entrenamiento en fútbol-base: diferencias entre dos meses durante el periodo competitivo en la categoría sub-19. SportisSci J, 5 (1), 30-52. DOI: https://doi.org/10.17979/sportis.2019.5.1.3469

http://revistas.udc.es/
} 
Artículo Original. Análisis de las tareas de entrenamiento en fútbol-base: diferencias entre dos meses durante el periodo competitivo en la sided categoría sub-19. Vol. V, nº. 1; p. 30-52, enero 2019. A Coruña. España ISSN 2386-8333

https://doi.org/10.1016/j.humov.2014.10.005

48. Turner, A.P., y Martinek, T.J. (1999). An investigation into teaching games for understanding: Effects on skill, knowledge, and game play. Research Quarterly for Exercise and $\quad$ Sport, $286-296 . \quad$ DOI: http://doi.org/10.1080/02701367.1999.10608047

49. Villarejo, D., Ortega, E., Gómez, M. A., y Palao, J. (2014). Design, validation and reliability of an observational instrument for ball possessions in rugby unión. International Journal of Performance Analysis, 14(3), 896-908. DOI: http://doi.org/10.1080/24748668.2014.11868771

\section{Agradecimientos}

Trabajo desarrollado dentro del Grupo de Optimización del Entrenamiento y Rendimiento Deportivo (G.O.E.R.D.) de la Facultad de Ciencias del Deporte de la Universidad de Extremadura.

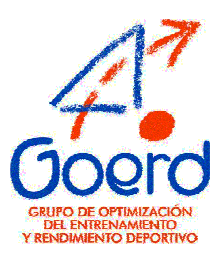

Este trabajo ha sido parcialmente subvencionado por la Ayuda a los Grupos de Investigación (GR15122) del Gobierno de Extremadura (Consejería de Empleo, Empresa e Innovación); con la aportación de la Unión Europea a través de los Fondos Europeos de Desarrollo Regional.

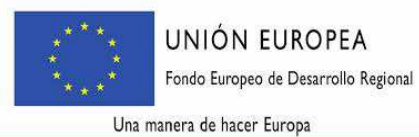

GOBIERNO DE EXTREMADURA

\footnotetext{
Para citar este artículo utilice la siguiente referencia: Gamonales Puerto, J.M.; Gómez Carmona, C.D.; León Guzmán, K.; García Santos, D.; Gamero Portillo, M.G.; Muñoz Jiménez, J. (2019). Análisis de las tareas de entrenamiento en fútbol-base: diferencias entre dos meses durante el periodo competitivo en la categoría sub-19. SportisSci J, 5 (1), 30-52. DOI: https://doi.org/10.17979/sportis.2019.5.1.3469 http://revistas.udc.es/
} 